\title{
Development and Validation of a New Simple Liquid Chromatographic Method for Determination of Coumarin and its 7-Hydroxy Metaboliteas a Marker of CytochromeP450 2A6 in Rats
}

\author{
Sundeep Bath ${ }^{1}$, Wael Ebied ${ }^{2}$, Hytham Ahmed ${ }^{3}$, Fawzy Elbarbry ${ }^{1,4 *}$ \\ ${ }^{1}$ School of Pharmacy, Pacific University, Hillsboro, OR, USA. \\ ${ }^{2}$ SEDICO Pharmaceuticals, Merck \& Co External Partner, 6th of October City, Cairo, Egypt. \\ ${ }^{3}$ Pharmaceutical Analysis department, Faculty of Pharmacy, Damanhour University, Damanhour, Egypt.
}

\author{
ARTICLE INFO \\ Article history: \\ Received on: 17/04/2015 \\ Revised on: 11/05/2015 \\ Accepted on: 22/05/2015 \\ Available online: 27/07/2015 \\ Key words: \\ HPLC, CYP2A6, Coumarin, \\ 7-hydroxy coumarin, \\ Resveratrol, Sulforaphane, \\ Thymoquinone, metabolism, \\ rats
}

\begin{abstract}
Objective: In this study, a simple and reliable high performance liquid chromatographic method with UV detection was developed and validated for rapid determination of coumarin hydroxylase activity in rat hepatic microsomes. Materials and Methods: The chromatographic separation was achieved using Zorbax Eclipse XDB C18 column $(150 \times 4.6 \mathrm{~mm}, 5 \mu \mathrm{m})$, which was kept at $40^{\circ} \mathrm{C}$. The isocratic mobile phase consisted of methanol and $1 \%$ glacial acetic acid mixture $(35: 65, \mathrm{v} / \mathrm{v})$ with a flow rate of $0.6 \mathrm{ml} / \mathrm{min}$. The effluent was monitored at $320 \mathrm{~nm}$ using photodiode array detector (PDA). 6-hydroxychlorzoxazone (6-OH CZX) was used as internal standard. Results and Conclusion: The method exhibited good linearity $\left(R^{2}>0.999\right)$ for both coumarin $(\mathrm{COUM})$ and its 7-hydroxy metabolite (7-OH COUM) over the assayed concentration range (0.025-5.0 $\mu \mathrm{M})$ and demonstrated good intra-day and inter-day precision and accuracy (relative standard deviations and the deviation from predicted values were less than $15 \%$ ). The detection limits were 0.001 and $0.005 \mu \mathrm{M}$ for coumarin and 7hydroxycoumarin, respectively. This method was also successfully applied for studying the effect of three phytochemicals on hepatic CYP2A6 activity in rats.
\end{abstract}

\section{INTRODUCTION}

Cytochrome P450 enzymes consist of a superfamily of enzymes and plays a pivotal role in the metabolism of xenobiotics and synthesis of endogenous substrates (Schneider and Clark, 2013). The CYP2A6 enzyme is a primary member of the CYP subfamily and is predominantly expressed in the human liver (Oscarson, 2001). CYP2A6 is involved in the metabolism of several drugs including phenobarbital, dexamethasone and rifampin (Le et al., 2003). Additionally, CYP2A6 metabolizes or bioactivate many environmental toxins including coumarin, aflatoxin $\mathrm{B}$, nicotine and $N$-nitrosodiethylamine and tobaccospecific nitrosamines (Oscarson, 2001). Thus it is pertinent to study CYP2A6 metabolism in order to evaluate its clinical and toxicological significance. Coumarin is the most reliable and

\footnotetext{
* Corresponding Author

Fawzy Elbarbry, PhD, RPh

School of Pharmacy, Pacific University Oregon

Email: fawzy.elbarbry@pacificu.edu
}

utilized marker substrate for CYP2A6 and several studies have confirmed that more than $90 \%$ of coumarin 7-hydroxylation activity is due to CYP2A6 enzyme (Figure 1) (Kim et al., 2005; Yuan et al., 2002). There are several HPLC-UV detection methods for assaying coumarin 7-hydroxylation in the literature. Some of the limitations associated with these methods include: time consuming extraction and evaporation steps (Killard et al., 1996;Lutz et al., 2002;Soucek, 1999), lengthy retention times (Waxman and Chang, 2006)and utilizing a complex mobile phase with expensive column such as monolithic silica rod (Lutz et al., 2002). Several animal studies and human clinical trials demonstrate that consumption of certain herbal remedies such as Resveratrol (RES) (Kulkarni and Canto, 2014), Sulforaphane (SF)(Elbarbry et al., 2014), and Thymoquinone (TQ)(Ragheb et al., 2009; Ragheb et al., 2008) decreases morbidity associated with chronic diseases. Accordingly, it is expected to see increased consumption of food and dietary sources of these herbal remedies. If widespread consumption of RES, SF, and TQ is expected, then there should be some understanding of how they may affect drug metabolizing enzymes. 
This study was undertaken to develop an accurate, simple and sensitive HPLC method to measure coumarin hydroxylase activity as a marker of CYP2A6 activity then apply the method to investigate the potential effect of RES, SF, and TQ on hepatic CYP2A6 activity in Spontaneously Hypertensive Rat (SHR) model.

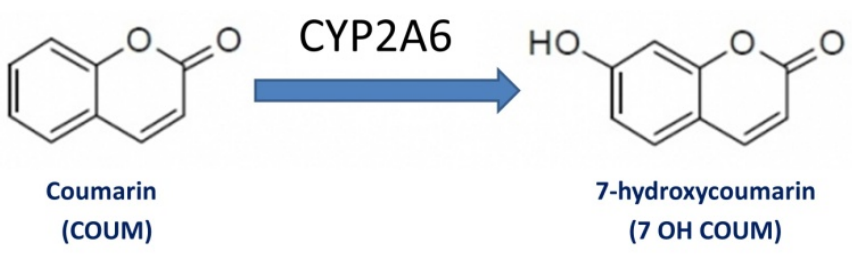

Fig. 1: CYP2A6-mediated COUM hydroxylation to 7-OH COUM.

This specific animal model of hypertension is currently used in our lab to investigate the antihypertensive effect of certain phytochemicals. The importance of this study is emphasized by the findings that certain phytochemicals in vegetables and fruits have the capacity to modulate drug metabolizing enzymes and significantly affect pharmacokinetics, and hence pharmacodynamics, of concomitantly administered medications.

\section{EXPERIMENTAL}

\section{Materials}

\section{Chemicals and reagents}

Coumarin (COUM), 7-hydroxycoumarin (7-OH COUM), 6-hydroxychlorzoxazone (6-OH CZX), thymoquinone (TQ) and all chemicals used for microsomal preparation, determination of microsomal protein content, and enzyme assays were purchased from Sigma-Aldrich (ST. Louis, MO).

Purified sulforaphane (SF) was obtained from LKT laboratories (St. Paul, MN). Resveratrol (RES) was purchased from TCI (Portland, OR). Acetonitrile and methanol were HPLC grade and obtained from Fisher Scientific (Pittsburg, PA). A Milli Q Synthesis (Millipore, Bedford, MA) water purification system provided purified deionized water. All other chemicals used were analytical grade.

\section{Animals}

Male 14-week-old SHR were obtained from Charles River Laboratories (Wilmington, MA). All animals were maintained under controlled housing conditions of light (6 AM-6 PM) with temperature $\left(22{ }^{\circ} \mathrm{C}\right)$ and received standard laboratory chow and water ad libitum. All rats were allowed at least 1 week to become acclimated to the housing conditions and ensure steady and reliable blood pressure readings before use in experiments. All procedures were approved by the Institutional Animal Care and Use Committee of Pacific University and Oregon Health \& Science University. Animals were randomly divided into 7 groups of 5 animals each. One group served as the control group, whereas the remaining groups were provided with either low dose (20 $\mathrm{mg} / \mathrm{kg}$ ) or high dose (40 mg/kg) of SF, RES, or TQ in the drinking water for 8 weeks. Preliminary stability studies using LC-MS indicated that RES, SF, and TQ solutions are stable in water at room temperature for at least 5 days (data not shown). Fresh solutions were made from stock twice a week. The selection of RES, SF, or TQ doses was based on preliminary studies in our laboratory and previous studies (Elbarbry et al., 2014); Kulkarni, 2014).

\section{Methods \\ Preparation of Hepatic Microsomes}

Hepatic microsomes were prepared as described previously (Elbarbry et al., 2012). Briefly, $0.5 \mathrm{~g}$ of liver was homogenized in $2 \mathrm{~mL}$ homogenization buffer $(50 \mathrm{mM}$ Tris buffer, $150 \mathrm{mM} \mathrm{KCl}, 0.1 \mathrm{mM}$ dithiothreitol, 1mM EDTA, 20\% glycerol, and $0.1 \mathrm{mM}$ phenylmethyl sulfonylfluoride).

The homogenate was centrifuged at $9000 \times g$ for $30 \mathrm{~min}$ in a Beckman L8-55 Ultracentrifuge (Palo Alto, CA, USA). The supernatant was carefully transferred to clean ultracentrifuge tubes and centrifuged at $100,000 \times \mathrm{g}$ for $30 \mathrm{~min}$. The pellet was washed in $2 \mathrm{~mL}$ of $150 \mathrm{mM} \mathrm{KCl}$ and centrifuged again at $100,000 \times \mathrm{g}$ for 30 min. The pellet was resuspended in $2 \mathrm{~mL}$ of $0.25 \mathrm{M}$ sucrose solution and $400 \mu \mathrm{L}$ aliquots were transferred to cryogenic microcentrifuge tubes $(1.5 \mathrm{~mL})$. Microsomal suspensions were stored at $-80{ }^{\circ} \mathrm{C}$ until use.

\section{Determination of Microsomal Protein Content}

Microsomal protein concentrations were determined by the method of Lowry et al.(Lowry et al., 1951) using bovine serum albumin as the standard. Absorbance was measured at $750 \mathrm{~nm}$ on a Synergy $2^{\circledR}$ micro-plate reader using Gen5 Software (BioTek, Winooski, VT).

\section{Chromatographic Conditions}

A Shimadzu LC 20A HPLC system (Maryland, USA) consisting of an auto-sampler, a degasser, a binary pump, a model SPD-20A photodiode array detector. Chromatographic separation was carried out on Zorbax Eclipse $\mathrm{XDB} \mathrm{C}_{18}$ column $(150 \times 4.6$ mm I.D., $5 \mu \mathrm{m}$ particle size).

The column was kept at $40^{\circ} \mathrm{C}$. The isocratic mobile phase consisted of methanol and 1\% glacial acetic acid mixture (35:65, $\mathrm{v} / \mathrm{v}$ ) was run at a flow rate of $0.6 \mathrm{ml} / \mathrm{min}$. Absorbance was monitored at $320 \mathrm{~nm}$. This wavelength was found adequate to monitor COUM, 7-OHCOUM and 6-OHCZX as indicated by using the PDA detector.

\section{Preparation of Standard Solutions and Calibration Standards}

COUM and 7-OH COUM were dissolved in dimethyl sulfoxide (DMSO) to prepare stock solutions $(10 \mathrm{mM})$ each. The stock solutions were stored at $-20^{\circ} \mathrm{C}$ where analytes were found to be stable for at least 8 weeks. Using these stock solutions, calibration standards were prepared in drug-free and heatinactivated rat hepatic microsomes at concentrations of $0,0.025$, $0.05,0.1,0.25,0.50,1.0,2.5$, and $5.0 \mu \mathrm{M}$ of each analyte. Three 
quality control (QC) samples were prepared by spiking drug-free rat hepatic microsomes with COUM and 7-OH COUM for method validation studies.

These QC samples were prepared to contain $0.25 \mu \mathrm{M}$ (QC1); $1.0 \mu \mathrm{M}(\mathrm{QC2})$; and $2.5 \mu \mathrm{M}(\mathrm{QC} 3)$ of each analyte. A working internal standard (IS) solution was prepared by diluting 6OH CZX stock solution $(1 \mathrm{mM})$ in methanol to obtain $100 \mu \mathrm{M}$.

\section{Microsomal Incubation}

7-Hydroxylase activity was determined by quantification of 7-OH COUM formation rate in rat hepatic microsomes. Preliminary experiments were conducted to determine linear metabolite formation kinetics with respect to COUM concentration, incubation time and microsomal protein concentration. Microsomal incubation mixtures consisted of COUM $(20 \mu \mathrm{M}), 0.4 \mathrm{mg} / \mathrm{ml}$ liver microsomal protein, $2 \mathrm{mM}$ $\mathrm{MgCl}_{2}, 1 \mathrm{mM} \mathrm{NADPH}$, and $50 \mathrm{mM}$ phosphate buffer, $\mathrm{pH} 7.4$, in a final volume of $0.5 \mathrm{ml}$. After a pre-incubation period of $3 \mathrm{~min}$ at $37^{\circ} \mathrm{C}$, the reaction was started by addition of NADPH and incubated at $37^{\circ} \mathrm{C}$ for $15 \mathrm{~min}$ in a shaking water bath. The reaction was terminated by addition of $50 \mu \mathrm{L}$ ice-cold $50 \%$ tri-chloroacetic acid as described before(Yuan et al., 2002). Metabolite formation rate was calculated by dividing the amount of the metabolites formed by the incubation time and microsomal protein content (nmol/min/mg).

\section{Sample Preparation}

To $200 \mu \mathrm{L}$ calibration standards, QC samples, or microsomal incubation mixtures, $50 \mu \mathrm{L}$ of the working internal standard solution was added. The mixtures were vortex mixed for 20 seconds. After centrifugation at $10,000 \times g$ in an Eppendorf microcentrifuge (Model $5417 \mathrm{C}$, Brinkmann instruments, Westbury, NY, USA) for $15 \mathrm{~min}, 20 \mu \mathrm{L}$ of the supernatant was injected directly onto the analytical column for immediate HPLC analysis.

\section{Method Validation}

Method validation procedures were performed according

to FDA guidelines

(http://www.fda.gov/downloads/drugs/guidancecomplianceregulat oryinformation/guidances/ucm070107.pdf, accessed Dec. 1, 2014) and as explained previously (Elbarbry et al., 2009;Elbarbry and Shoker, 2007) to evaluate the suitability of the method for the quantitative determination of COUM and its 7-OH metabolite, in rat hepatic microsomes. Specificity was tested by analysis of five independent drug-free rat hepatic microsomes supplemented only with IS to ensure the absence of endogenous compounds with the same retention times at the analytes of interest. The linearity of the method was evaluated by processing eight-point calibration curves range $0.025-5.0 \mu \mathrm{M}$ on six different days. The peak height ratios of each analyte to the internal standard were plotted against the analyte's nominal concentration. A linear least-squares regression analysis was conducted to determine slope, intercept and coefficient of determination $\left(R^{2}\right)$ to demonstrate linearity of the method. The lower limit of detection (LLOD) was defined as the lowest detectable concentration, taking into consideration a signalto-noise $(\mathrm{S} / \mathrm{N})$ ratio of 3. Lower limit of quantification (LLOQ) was determined as the lowest concentration at which the precision, expressed as \% coefficient of variation (CV), is less than $20 \%$, accuracy is $80-120 \%$ and $\mathrm{S} / \mathrm{N}$ ratio is at least 10 . The accuracy and precision of the proposed method were determined by analysis of the QC samples. The intra-day accuracy and precision were assessed from the results of five replicate analyses of QC samples on a single assay day. The inter-day accuracy and precision were determined from the same QC samples analyzed on 6 consecutive days. Accuracy $(\%)$ is expressed as [(calculated amount/predicted amount) $x 100]$. The mean value should be within $15 \%$ of the predicted value except at LLOQ, where it should not deviate by more than $20 \%$. Precision is expressed as \% CV, and it should not exceed $15 \%$ except for the LLOQ, where it should not exceed $20 \%$.

Stability of QC samples was evaluated after long-term (frozen at $-20^{\circ} \mathrm{C}$ ) and short-term (bench top, room temperature) storage, and after going through three freeze and thaw cycles. The stability of stock solutions of COUM, 7-OH COUM and IS was also evaluated as described below:

i. Freeze and Thaw Stability: Three aliquots of each QC sample were stored at $-20^{\circ} \mathrm{C}$ for 24 hours and thawed unassisted at room temperature. The freeze-thaw cycle was repeated two more times, and then analyzed on the third cycle.

ii. Short-Term Temperature Stability: Three aliquots of each QC sample were thawed at room temperature and analyzed at 4 and 12 hours (based on the expected duration that samples will be maintained at the HPLC auto-sampler during analysis)

iii. Long-Term Temperature Stability: Three aliquots of each QC sample were kept at $-20^{\circ} \mathrm{C}$ for two weeks (the estimated time between the date of first sample collection and the date of last sample analysis).

iv. Stock Solution Stability: The stability of stock solutions of COUM, 7-OH COUM, and the internal standard were evaluated at room temperature for 6 hours and at $-20^{\circ} \mathrm{C}$ for 2 weeks.

\section{Data Analysis}

Data are reported as mean \pm SD. Difference in CYP2A6 activity among groups was assessed by one-way Analysis of Variance (ANOVA) with Turkey's post-hoc test for pair-wise multiple comparisons. Statistical analysis was conducted using GraphPad Prism 5.0 (GraphPad Software Inc., San Diego, CA).

\section{RESULTS AND DISCUSSION}

As studying the effect of herbal remedies on the activity of drug metabolizing enzymes is a routine experiment in our laboratory, development of simple, cost-effective, accurate, fast and sensitive HPLC methods is a key. Due to its clinical and toxicological relevance, CYP2A6 is one of the most important P450 enzymes. Hydroxylation of COUM to 7-OH COUM has 
been used as a marker for CYP2A6 in biological samples (Soucek, 1999). In our preliminary experiments using published fluorescence-based HPLC methods, selectivity was an issue as some of the studied herbal remedies or their metabolites co-elute with COUM or 7-OH COUM. Additionally, accuracy and precision were frequently below the accepted standards and a use of IS in the HPLC analysis was needed to improve them. Therefore, we described in this article a new UV-based HPLC assay for COUM and its 7-OH metabolite as a marker of CYP2A6. After trying several mobile phase compositions and different columns, methanol and $1 \%$ glacial acetic acid (35/65) was chosen as a mobile phase and Zorbax Eclipse $\mathrm{XDB} \mathrm{C}_{18}$ was selected as a stationary phase on the basis of shorter retention time, symmetric peaks, good resolution, and excellent selectivity. 6-OH CZX is structurally-similar to COUM and its metabolite, and was found to be a good internal standard in this method. Under the chromatographic conditions explained in the methods section, 6$\mathrm{OH} \mathrm{CZX}, 7-\mathrm{OH}$ COUM, and COUM were eluted and well separated at retention times $6.2,7.5$, and 10.9 min respectively (Figure 2a).
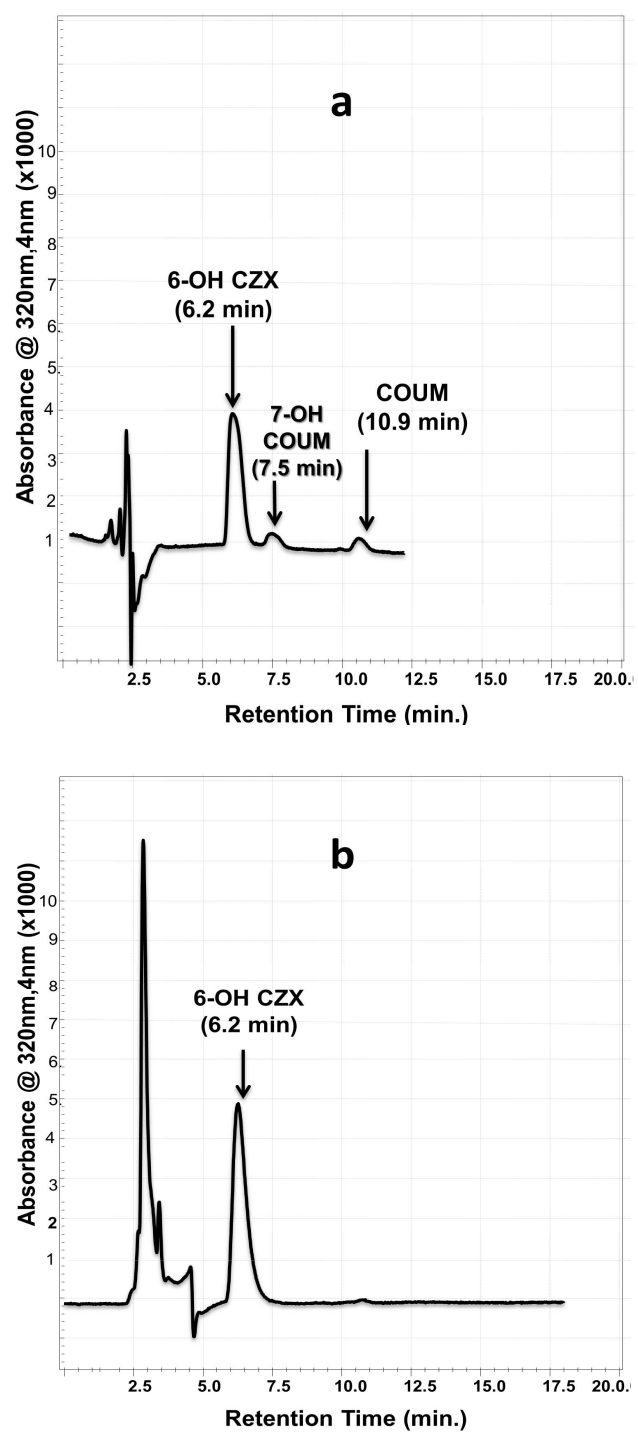

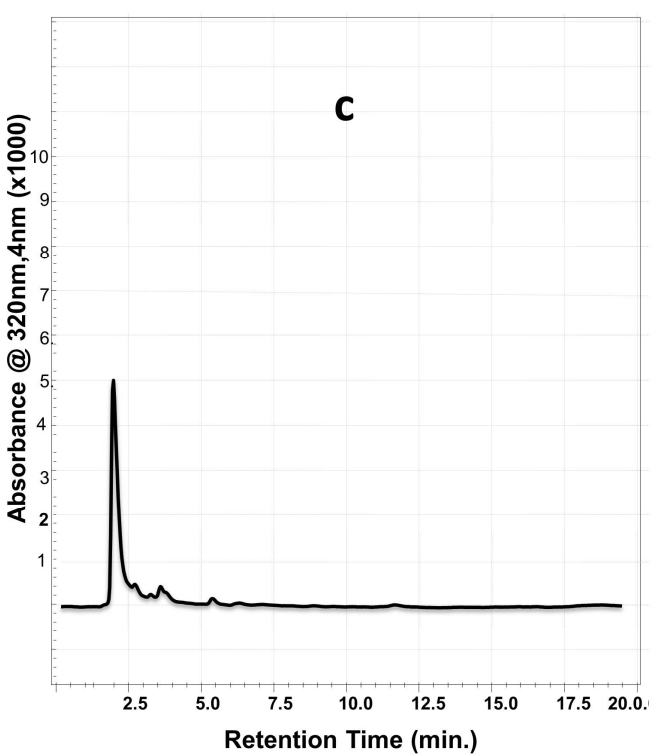

Fig. 2: Typical HPLC chromatograms of COUM and its metabolite (7-OH COUM) in rat hepatic microsomes samples. (a) Blank rat hepatic microsomes sample spiked with internal standard (6-OH CZX), 7-OH COUM $(0.025 \mu \mathrm{M})$, and COUM $(0.025 \mu \mathrm{M})$. (b) Blank rat hepatic microsomes sample spiked only with the internal standard $(20 \mu \mathrm{M})$ shows no interfering peaks at retention times of COUM or 7-OH COUM. (c) Blank rat hepatic microsomes sample without any spiking shows no interfering peaks at retention times of the analytes or the internal standard.

\section{Method Validation Selectivity}

Selectivity of the proposed analytical method was indicated by the absence of any endogenous interference at retention times of peaks of interest as evaluated by chromatograms of blank rat hepatic microsomes (Figure 2c) and blank rat hepatic microsomes spiked only with the internal standard (Figure 2b).

\section{Linearity}

Calibration curves were constructed by plotting peak height ratios of the analyte to IS versus concentration of the analyte. The calibration curves of COUM and its metabolite in rat hepatic microsomes were linear over concentration ranges of 0.025-5 $\mu \mathrm{M}$ (Table 1). The coefficient of determination $\left(\mathrm{R}^{2}\right)$ was greater than 0.999 in all calibration curves $(n=6)$. The mean regression equations were $y=1.212 x-0.0221$ for 7-OH COUM and $y=2.71 x-0.0197$ for COUM. Lower limit of detection (LLOD) and quantitation (LLOQ) were determined based upon Signal-to-Noise (S/N) ratio of 3 and 10, respectively. Spiked rat hepatic microsomes (for COUM and its metabolite) were assayed for all analytes in decreasing concentration. Chromatograms from the assay of blank hepatic microsomal samples were used for the establishment of the background noise. LLOD of COUM and 7-OH COUM was 0.001 and $0.005 \mu \mathrm{M}$, respectively. However, LLOQ for both analytes, where maximal intra- and inter-day variation in accuracy and precision is $20 \%$, was $0.025 \mu \mathrm{M}$. The range and sensitivity level of calibration curves in our method were sufficient to measure coumarin hydroxylase activity in rat hepatic microsomes. 
Table. 1: Linearity data for the quantification of COUM and its metabolite, 7-OH COUM in in rat hepatic microsomes by the proposed HPLC method

\begin{tabular}{|c|c|c|c|c|c|c|c|c|c|}
\hline & \multicolumn{2}{|c|}{ Intercept } & \multicolumn{2}{|c|}{ Slope } & \multicolumn{2}{|c|}{$\mathbf{R}^{2}$} & \multirow{2}{*}{$\frac{\text { LLOQ }}{(\mu \mathrm{M})}$} & \multirow{2}{*}{$\frac{\text { LLOD }}{(\mu \mathrm{M})}$} & \multirow{2}{*}{$\begin{array}{r}\text { Linearity Range } \\
(\mu \mathrm{M})\end{array}$} \\
\hline & Average* & SD & Average* & SD & Average* & SD & & & \\
\hline 7-OH COUM & -0.0221 & 0.018 & 1.22 & 0.287 & 0.99960 & 0.000274 & 0.025 & 0.005 & $0.025-5$ \\
\hline $\begin{array}{l}\text { COUM } \\
*_{n}=6\end{array}$ & -0.0197 & 0.023 & 2.71 & 0.391 & 0.99964 & 0.000232 & 0.025 & 0.001 & $0.025-5$ \\
\hline
\end{tabular}

Table. 2: Intra-day $(\mathrm{n}=5)$ and inter-day (6 consecutive days) accuracy and precision data for the quantitation of COUM and its metabolite, 7-OH COUM in in rat hepatic microsomes by the proposed HPLC method.

\begin{tabular}{|c|c|c|c|c|c|c|c|c|c|}
\hline & \multirow{2}{*}{$\begin{array}{c}\text { Nominal Conc. } \\
(\mu \mathrm{M})\end{array}$} & \multicolumn{3}{|c|}{ Intra-day $(n=5)$} & \multicolumn{5}{|c|}{ Inter-day $(\mathrm{n}=6)$} \\
\hline & & Average & SD & Accuracy \% & CV\% & Average & SD & Accuracy \% & CV\% \\
\hline \multirow[t]{3}{*}{ 7-OH COUM } & 0.25 & 0.281 & 0.022 & 112 & 7.74 & 0.268 & 0.022 & 107 & 8.18 \\
\hline & 1 & 0.986 & 0.074 & 98.5 & 7.55 & 1.047 & 0.052 & 104 & 5.02 \\
\hline & 2.5 & 2.652 & 0.144 & 106 & 5.43 & 2.823 & 0.148 & 112 & 5.23 \\
\hline \multirow[t]{3}{*}{ COUM } & 0.25 & 0.254 & 0.015 & 102 & 5.87 & 0.259 & 0.025 & 103 & 9.67 \\
\hline & 1 & 1.04 & 0.05 & 104 & 7.12 & 1.085 & 0.073 & 108 & 6.77 \\
\hline & 2.5 & 2.54 & 0.107 & 101 & 7.17 & 2.505 & 0.107 & 100 & 4.28 \\
\hline
\end{tabular}

Table. 3: Stability of COUM, 7-OH COUM, and 6-OH CZX in solution under different storage and handling conditions.

\begin{tabular}{|c|c|c|c|c|c|c|c|c|c|c|c|c|c|c|}
\hline \multicolumn{3}{|c|}{ Freeze-Thaw Stability } & \multicolumn{4}{|c|}{ Short-Term Stability } & \multicolumn{2}{|c|}{$\begin{array}{l}\text { Long-Term } \\
\text { Stability }\end{array}$} & \multicolumn{6}{|c|}{ Stock Solution Stability } \\
\hline & & & \multicolumn{2}{|c|}{ After $4 \mathrm{hr}$} & \multicolumn{2}{|c|}{ After 12 hr } & \multirow[b]{2}{*}{ COUM } & \multirow[b]{2}{*}{$\begin{array}{l}\text { 7-OH } \\
\text { COUM }\end{array}$} & \multicolumn{3}{|c|}{ Room Temp. for $6 \mathrm{hr}$. } & \multicolumn{3}{|c|}{$-20^{\circ} \mathrm{C}$ for 2 weeks } \\
\hline & COUM & $\begin{array}{l}\text { 7-OH } \\
\text { COUM }\end{array}$ & COUM & $\begin{array}{l}\text { 7-OH } \\
\text { COUM }\end{array}$ & COUM & $\begin{array}{l}\text { 7-OH } \\
\text { COUM }\end{array}$ & & & COUM & $\begin{array}{l}\text { 7-OH } \\
\text { COUM }\end{array}$ & $\begin{array}{l}\text { 6-OH } \\
\text { CZX }\end{array}$ & COUM & $\begin{array}{l}\text { 7-OH } \\
\text { COUM }\end{array}$ & $\begin{array}{l}\text { 6-OH } \\
\text { CZX }\end{array}$ \\
\hline QC1 & $95.5 \pm 2.5$ & $101 \pm 2.5$ & $99.5 \pm 2$ & $100.5 \pm 3$ & $97.5 \pm 3$ & $101.5 \pm 1.5$ & $98 \pm 3.5$ & $102 \pm 3$ & $97 \pm 4.5$ & $104 \pm 1.5$ & $105 \pm 4.5$ & $104 \pm 2.5$ & $100 \pm 2.51$ & $100 \pm 2.5$ \\
\hline QC2 & $99 \pm 2$ & $102 \pm 1.5$ & $97.5 \pm 1.5$ & $102.5 \pm 2$ & $99 \pm 2.5$ & $97 \pm 3$ & $102 \pm 3$ & $97 \pm 1.5$ & & & & & & \\
\hline QC3 & $98 \pm 3.5$ & $98 \pm 3$ & $101.5 \pm 2$ & $99.5 \pm 2.5$ & $103 \pm 3$ & $95 \pm 2.5$ & $95 \pm 1.5$ & $100 \pm 5$ & & & & & & \\
\hline
\end{tabular}

\section{Accuracy and Precision}

To determine intra-day and inter-day accuracy and precision of the assay, replicate set ( $\mathrm{n}=5$ and 6 , respectively) of three concentrations (low, medium and high) of each analyte in rat hepatic microsomes were analyzed. Accuracy and precision were calculated as described in the materials and methods section and results are summarized in Table 2 . The intra-assay and inter-assay precision for COUM and its metabolite were less than $10 \%$ in all cases. Blindly assayed QC samples of both COUM and 7-OH COUM show an average intra-day and inter-day accuracy in the range of 98-112\% (Table 2). These data showed that the proposed method was both accurate and precise for the determination of COUM and its metabolite in rat hepatic microsomes.

\section{Stability}

To evaluate the stability of COUM, 7-OH COUM, and IS under storage and handling conditions, separate QC standards, covering the low $(0.05 \mu \mathrm{M})$, medium $(1 \mu \mathrm{M})$, and high ranges $(2.5 \mu \mathrm{M})$, were prepared for different temperatures and times.

The accuracy of COUM and 7-OH COUM obtained after three freeze-thaw cycles, short-term storage (4-12 hr.) at room temperature, and long-term storage (2 weeks) at $-20^{\circ} \mathrm{C}$ was $95-105 \%$, as shown Table 3. Additionally, the standard stock solution of both analytes and IS were found to be stable for at least $6 \mathrm{hr}$. at room temperature and for 2 weeks at $-20^{\circ} \mathrm{C}$ (Table 3). The stability of both analytes and IS was consistent with other reports (Kim et al., 2005;Soucek, 1999).

\section{Application of the Proposed Method}

COUM is extensively metabolized by CYP2A6 isozyme to 7-OH COUM (Yuan et al., 2002). Accordingly, COUM hydroxylase is a commonly used marker for CY2A6 activity. Our validated HPLC-UV method is adequately sensitive, accurate, and precise to evaluate COUM hydroxylase activity in rat hepatic microsomes.
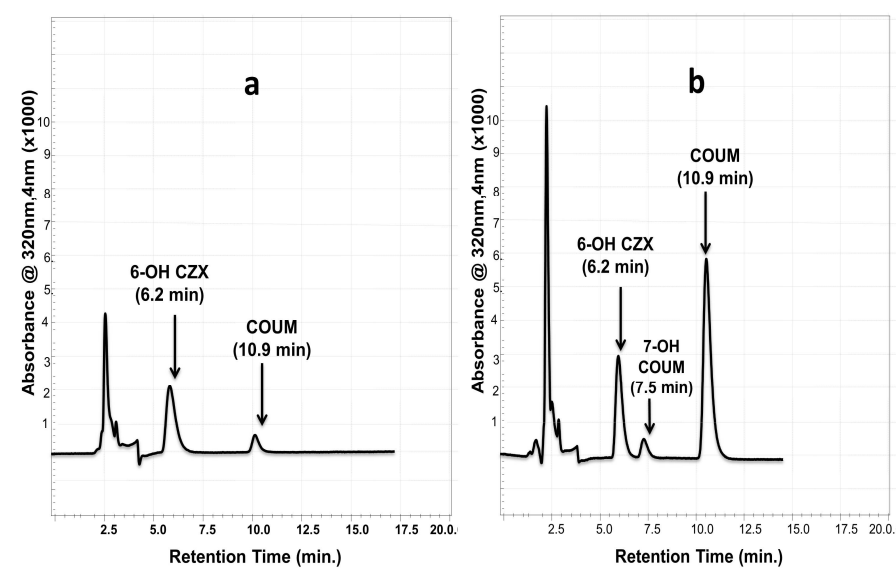

Fig. 3: Representative HPLC chromatograms of rat hepatic microsomes after incubation with COUM $(20 \mu \mathrm{M})$ in the absence (a) and presence (b) of $\mathrm{NADPH}$. Incubation conditions are explained in the methods section

Figures $3 \mathrm{a}$ and $3 \mathrm{~b}$ indicated that COUM hydroxylation was exclusively mediated by microsomal CYP enzymes and required NADPH for electron transfer. Recent research in our lab has indicated that certain phytochemicals such as sulforaphane (SF), resveratrol (RES), and thymoquinone (TQ) modulate the CYP-mediated metabolism of arachidonic acid in SHR model, a mechanism through which these phytochemicals protect SHR rats against the progressive rise in blood pressure (Elbarbry et al., 2014).

In the current study, we have utilized the developed HPLC method to investigate the effect of these phytochemical on 
CYP2A6 activity through measuring the rate of 7-OH COUM formation in hepatic microsomes of SHR following administration of different doses of these phytochemicals in their drinking water. As shown in Figure 4, no significant effect of either SF or TQ was observed on CYP2A6 activity.

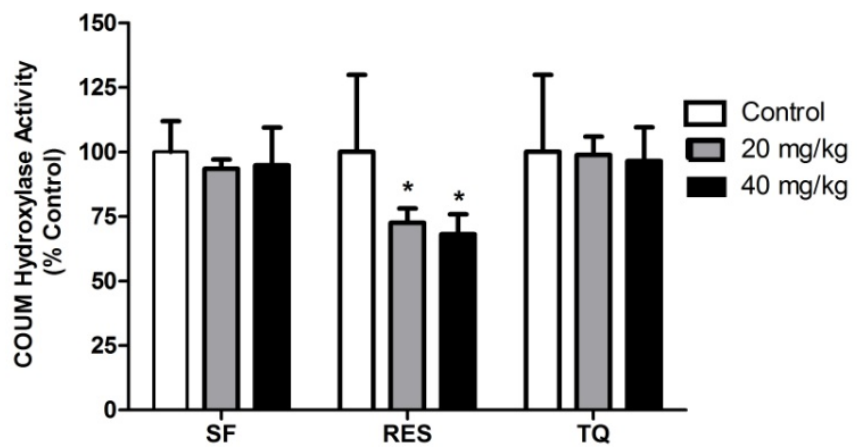

Fig. 4: Effect of administration of dietary doses of sulforaphane (SF), Resveratrol (RES), and Thymoquinone (TQ) on hepatic microsomal CYP2A6 activity in SHR rats. Male SHR rats $(\mathrm{n}=5)$ were administered $20 \mathrm{mg} / \mathrm{kg}$ or 40 $\mathrm{mg} / \mathrm{kg}$ of SF or RES or TQ in their drinking water for 8 weeks and livers were harvested at the end of the study. Activities of CYP2A6 enzyme was determined using the proposed HPLC method and as explained in the method section. All groups were compared using one-way ANOVA followed by multiple comparisons. *Significant difference from control with $P<0.05$. On the other hand, $20 \mathrm{mg} / \mathrm{kg}$ and $40 \mathrm{mg} / \mathrm{kg}$ RES treatment resulted in $28 \%$ and $33 \%$ inhibition of CYP2A6 activity, respectively. This CYP enzyme is the principal enzyme responsible for the oxidation of nicotine. Additionally, it is also involved in the clearance of several pharmaceuticals and carcinogens (Le et al., 2003). Inhibition of CYP2A6 activity by RES could pose a risk of drugherb interactions and a caution should be exercised in prescribing compounds metabolized by CYP2A6 enzyme in patients who are taking RES or RES-congaing natural products. On the other hand, RES may offer protection against carcinogens such as aflatoxin B1 and nitrosamines that are bio-activated by CYP2A6 (Higashi et al., 2007; Oscarson, 2001).

\section{CONCLUSION}

In this study, we described an HPLC-UV method for the simultaneous determination of COUM and its 7-OH metabolite as a surrogate marker for CYP2A6 enzyme activity. This new method offers the advantages of simplicity, specificity, precision, adequate sensitivity, and low sample volume. We successfully applied the method to determine CYP2A6 activity in rat hepatic microsomes and to investigate the effect of three commonly used phytochemicals SF, RES, and TQ on CYP2A6 activity in a rat model of hypertension.

\section{ACKNOWLEDGEMNTS}

We thank Anik Amin and Margarita Can for microsomal preparation. This work was supported in part by the Medical Research Foundation of Oregon (MRF), Collins Medical Trust, Faculty Development Grant from Pacific University, Oregon and Dean's Incentive Grant from Pacific University School of Pharmacy, Oregon (F.E.).

\section{REFERENCES}

Elbarbry F, Attia A, Shoker A. Validation of a new HPLC method for determination of midazolam and its metabolites: application to determine its pharmacokinetics in human and measure hepatic CYP3A activity in rabbits. J Pharm Biomed Anal 2009; 50: 987-993.

Elbarbry F, Ragheb A, Marfleet T, Shoker A. Modulation of hepatic drug metabolizing enzymes by dietary doses of thymoquinone in female New Zealand White rabbits. Phytother Res 2012; 26: 1726-1730.

Elbarbry F, Vermehren-Schmaedick A, Balkowiec A. Modulation of arachidonic Acid metabolism in the rat kidney by sulforaphane: implications for regulation of blood pressure. ISRN Pharmacol 2014; 2014: 683508.

Elbarbry FA, Shoker AS. Liquid chromatographic determination of mycophenolic acid and its metabolites in human kidney transplant plasma: pharmacokinetic application. J Chromatogr B Analyt Technol Biomed Life Sci 2007; 859: 276-281.

Higashi E, Nakajima M, Katoh M, Tokudome S, Yokoi T. Inhibitory effects of neurotransmitters and steroids on human CYP2A6. Drug Metab Dispos 2007; 35: 508-514.

Killard AJ, O'Kennedy R, Bogan DP. Analysis of the glucuronidation of 7-hydroxycoumarin by HPLC. J Pharm Biomed Anal 1996; 14: 1585-1590.

Kim D, Wu ZL, Guengerich FP. Analysis of coumarin 7 hydroxylation activity of cytochrome P450 2A6 using random mutagenesis. J Biol Chem 2005; 280: 40319-40327.

Kulkarni SS, Canto C. The molecular targets of Resveratrol. Biochim Biophys Acta 2014

Le GA, Dreano Y, Lucas D, Berthou F. Diversity of selective environmental substrates for human cytochrome P450 2A6: alkoxyethers, nicotine, coumarin, N-nitrosodiethylamine, and Nnitrosobenzylmethylamine. Toxicol Lett 2003; 144: 77-91.

Lowry OH, ROSEBROUGH NJ, FARR AL, RANDALL RJ. Protein measurement with the Folin phenol reagent. J Biol Chem 1951; 193: 265-275.

Lutz ES, Markling ME, Masimirembwa CM. Monolithic silica rod liquid chromatography with ultraviolet or fluorescence detection for metabolite analysis of cytochrome P450 marker reactions. J Chromatogr B Analyt Technol Biomed Life Sci 2002; 780: 205-215.

Oscarson M. Genetic polymorphisms in the cytochrome P450 2A6 (CYP2A6) gene: implications for interindividual differences in nicotine metabolism. Drug Metab Dispos 2001; 29: 91-95.

Ragheb A, Attia A, Eldin WS, Elbarbry F, Gazarin S, Shoker A. The protective effect of thymoquinone, an anti-oxidant and antiinflammatory agent, against renal injury: a review. Saudi J Kidney Dis Transpl 2009; 20: 741-752.

Ragheb A, Elbarbry F, Prasad K, Mohamed A, Ahmed MS, Shoker A. Attenuation of the development of hypercholesterolemic atherosclerosis by thymoquinone. Int J Angiol 2008; 17: 186-192.

Schneider E, Clark DS. Cytochrome P450 (CYP) enzymes and the development of CYP biosensors. Biosens Bioelectron 2013; 39: 1-13.

Soucek P. Novel sensitive high-performance liquid chromatographic method for assay of coumarin 7-hydroxylation. J Chromatogr B Biomed Sci Appl 1999; 734: 23-29.

Waxman DJ, Chang TK. Spectrofluorometric analysis of CYP2A6-catalyzed coumarin 7-hydroxylation. Methods Mol Biol 2006; 320: 91-96.

Yuan R, Madani S, Wei XX, Reynolds K, Huang SM. Evaluation of cytochrome $\mathrm{P} 450$ probe substrates commonly used by the pharmaceutical industry to study in vitro drug interactions. Drug Metab Dispos 2002; 30: 1311-1319.

\section{How to cite this article:}

Sundeep Bath, Wael Ebied, Hytham Ahmed, Fawzy Elbarbry. Development and Validation of a New Simple Liquid Chromatographic Method for Determination of Coumarin and its 7 Hydroxy Metabolite as a Marker of Cytochrome P450 2A6 in Rats. J App Pharm Sci, 2015; 5 (07): 130-135. 